\title{
Evaluation de la diversité floristique de six clairières sur schiste dans le massif forestier de Boumba-Bek et Nki, Sud-Est Cameroun
}

\author{
Paul NOUPA ${ }^{1 *}$ et Bernard-Aloys NKONGMENECK ${ }^{2,3}$ \\ ${ }^{1}$ Union Internationale pour la Conservation de la Nature (UICN), B.P. 5506 Yaoundé, Cameroun. \\ ${ }^{2}$ Laboratoire de Systématique et d'Ecologie, Département de Biologie et de Physiologie Végétales, Université \\ de Yaoundé I, Cameroun. \\ ${ }^{3}$ Musée d'Ecologie du Millénium (MEM) de Yaoundé, B.P. 8038 Yaoundé, Cameroun. \\ *Auteur correspondant, Tel: (237) 9964 16 46,E-mail: paul.noupa@iucn.org
}

\section{RESUME}

Les clairières forestières qui représentent à peine $1 \%$ de la superficie de forêt dans le Bassin du Congo, sont des ouvertures naturelles ayant un effet sur la concentration de la faune de par son rôle de pâturage. Dans le massif de Boumba-Bek au Sud-Est Cameroun, l'étude de la diversité floristique de la végétation herbacée de six clairières forestières a été conduite par sondage en utilisant la méthode d'échantillonnage systématique. Afin d'évaluer la diversité des clairières, l'inventaire floristique de la strate herbacée était réalisé avec la méthode phytosociologique sigmatiste de Braun Blanquet et toutes les espèces végétales contenues dans le placeau inventorié de $1 \mathrm{~m}^{2}$ étaient comptées et enregistrées sur des fiches standard d'inventaire de la végétation. L'étude a révélé que 68 espèces d'herbacées appartenant à 53 genres réparties entre 26 familles colonisent les clairières étudiées. Cinq principales familles, Poaceae, Cyperaceae, Fabaceae, Commelinaceae, Melastomataceae représentent $60,3 \%$ du cortège végétal dont 39,7\% pour le seul peuplement graminoïde: Poaceae $(26,5 \%)$ et Cyperaceae $(13,2 \%)$. Le cortège végétal du milieu est plus diversifié que riche. Les inventaires ayant porté sur 1030 relevés ont montré que 924 de ces relevés, soit 89,7\%, sont diversifiés alors que 106 relevés, soit 10,3\%, sont riches. L'évaluation quantitative et qualitative par distribution de présence/fréquence a permis de mettre en évidence les ressemblances physionomiques entre les clairières et les différences entre les unités d'échantillonnage.

(C) 2008 International Formulae Group. All rights reserved.

Mots clés: Ecosystème, Clairières forestières, Végétation herbacée, Parc National de BoumbaBek, phytosociologie, Bassin du Congo.

\section{INTRODUCTION}

La présente étude est menée dans le massif forestier de Boumba-Bek et Nki dans le Sud-Est du Cameroun (Figure 1). Le massif de Boumba-Bek et Nki qui s'étend de $2^{\circ} 00^{\prime}$ à $3^{\circ} 00^{\prime} \mathrm{N}$ de latitude et $14^{\circ} 00^{\prime}$ à $15^{\circ} 30^{\prime} \mathrm{E}$ de longitude, est le dernier bloc forestier qui n'a pas été perturbé par l'exploitation forestière dans le Sud-Est du Cameroun du fait de sa position géographique. En effet, l'exploitation du bois dans la partie forestière du Cameroun remonte aux années 30 et n'a pas touché le massif de Boumba-Bek du fait de sa position coincée entre les fleuves Boumba et Dja, qui lui a garanti une configuration de refuge pour une importante population de faune et un « hot spot» pour la biodiversité de la région. Ekobo (1998) indique que la région supporte une importante biomasse animale dans la classe des grands mammifères et représente un des refuges de la faune mammalienne du pléistocène dans le monde. Pour cette raison, des actions ont été entreprises pour mener des études biologiques et socio-économiques en vue du classement d'une partie de ce massif en Parc National afin de lui donner un statut qui lui permette de demeurer une entité territoriale à vocation de conservation de la 


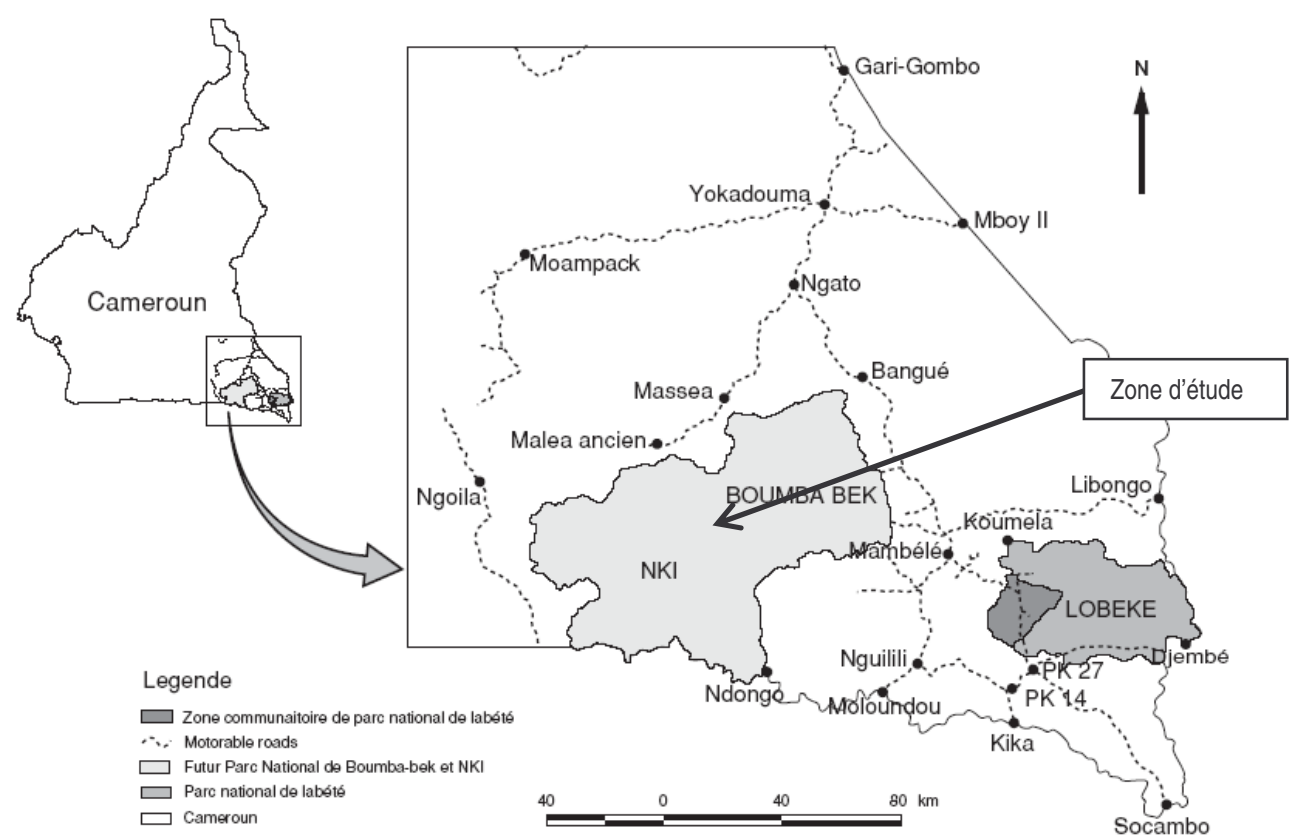

Figure 1: Localisation de la zone d'étude.

biodiversité et qui s'insère dans un réseau sous-régional d'unités de conservation représentatif de l'écosystème de forêt dense humide d'Afrique. Le Parc National de Boumba-Bek d'une superficie de 238.255 ha a été créé par Décret $N^{\circ} 2005 / 3284 / \mathrm{PM}$ du 6 octobre 2005 .

Dans le massif forestier de BoumbaBek et Nki (Sud-Est Cameroun) qui appartient au bloc camerounais de la forêt dense humide du Bassin du Congo, plusieurs auteurs ont classé les écosystèmes. Ainsi, Letouzey (1969) y a distingué une gamme d'écosystèmes qui vont de forêt ombrophiles type humide aux formations ligneuses parsemées. Nkongmeneck (1996) y a identifié plusieurs entités paysagères qui s'identifient davantage aux savanes à savoir :

- Les Clairières sèches,

- les Clairières humides,

- les Prairies sèches,

- les Prairies humides,

De nombreux auteurs (Nkongmeneck, 1996 ; Harris 1999, Noupa et al., 2002) ont reconnu l'individualité floristique des clairières forestières et ont relevé leur importance pour la conservation de la faune dans l'écosystème de la forêt dense du Sud-Est. Une étude sur l'influence des clairières forestières sur la répartition spatiale des grands mammifères dans la forêt dense du bassin du Congo conduite dans le même site a permis de savoir que les clairières forestières ont des concentrations des empreintes d'animaux quatre à six fois plus grande en leur centre que dans le reste du milieu environnant (Noupa et Nkongmeneck, 2008). Par ailleurs, la grande diversité des formations herbeuses en milieu forestier est relevée dans différents documents stratégiques de gestion des ressources naturelles dans le Bassin du Congo à l'instar des rapports sur la biodiversité (MIME, 2001 ; Anonyme, 1990) et malgré cela, force est de constater que très peu d'études ont été menées pour connaître la richesse de la diversité biologique et les potentialités de production des ces écosystèmes. Pourtant les clairières forestières supportent l'essentiel des besoins alimentaires de la population des herbivores sauvages de la région et constituent par ce fait les supports vitaux de la conservation de la richesse faunique des régions forestières. Letouzey (1985) a estimé que les écosystèmes 
de clairières toutes catégories confondues représentent seulement $1 \%$ de la superficie de forêt dans le Bassin du Congo et cette estimation est confirmée dans le rapport MIKE du site de Boumba-Bek (Bene Bene, 2004). Ces écosystèmes sont caractérisés par une formation végétale fermée dans laquelle dominent les plantes herbacées de plus de 80 $\mathrm{cm}$ de hauteur. Certaines sources indiquent des superficies plus grandes. Par exemple, au Congo, la «savane » occuperait 12 millions d'hectares, ce qui représenterait $35 \%$ du pays alors que la «savane» littorale couvre 300.000 hectares et représente $1 \%$ de la superficie du Pays (Mime, 2001). De toute évidence, l'insuffisance des connaissances sur une entité écologique importante pour la conservation de la biodiversité dans les massifs forestiers du Bassin du Congo est un handicap majeur pour l'aménagement des ressources fauniques, comme c'est le cas pour les aires protégées de Boumba-Bek et Nki. L'essentiel des préoccupations de connaissances sur ces clairières est relevé entre autres par les auteurs du document stratégique sur la biodiversité en RDC (Anonyme, 1990). En reconnaissant l'importance de ces écosystèmes, ces auteurs expriment les incertitudes sur les origines de ces formations et le mécanisme de leur maintien en état climaxique, ce qui se résume au problème de la dynamique et de la composition du couvert végétal de ces écosystèmes.

L'étude, menée dans six clairières du massif forestier de Boumba-Bek et Nki vise à contribuer à l'amélioration des connaissances sur la diversité floristique de ces écosystèmes afin de répondre à une partie des préoccupations dans la perspective de l'aménagement de la faune. En effet, du fait de la méconnaissance, les écosystèmes de clairière ont été par le passé peu considérés dans les processus de valorisation du milieu naturel et leurs rôles pour les équilibres biologiques ignorés. Par ailleurs, beaucoup de clairières forestières auraient été par le passé détruites par les populations pour installer les villages, les zones agricoles et les zones de chasse comme résultat de l'interférence de ces écosystèmes avec l'Homme. On pense que la plupart des villages installés dans le milieu forestier dans le Bassin du Congo, l'ont été dans les clairières forestières à cause de la facilité à les défricher ou à les dégager avec le feu, et surtout du fait de la fertilité de leurs sols qui les rend propices à l'agriculture (Encarta, 2000).

Avec l'évolution dans l'intérêt de la communauté nationale et internationale pour la conservation de la diversité faunique et la préservation des entités représentatives des habitats dans le Bassin du Congo, les clairières forestières présentent un intérêt majeur pour l'aménagement des Aires Protégées, ce qui justifie que cette étude contribue à une telle fin.

\section{MATERIEL ET METHODES Inventaires floristiques}

L'inventaire floristique qui a été conduite dans les clairières a utilisé à la fois les outils floristiques et écologiques. Il a été procédé à un inventaire floristique par sondage par la méthode d'échantillonnage systématique. Pour ce faire, dans chaque clairière étudiée, il a été matérialisé des transects de base séparées à équidistance les uns des autres de 100 mètres. Sur chaque transect de base, il a été délimité des parcelles de sondage et les parcelles consécutives étaient équidistantes de 50 mètres. Une fois localisée, cette parcelle qui avait $9 \mathrm{~m}^{2}$ de surface était systématiquement divisée en 9 sous parcelles ou quadras de $1 \mathrm{~m}^{2}$ désignés Lot 1 à Lot 9. Le relevé de la végétation était systématiquement conduit sur les lots 1, 3, 5, 7 , et 9 , soit au total $5 \mathrm{~m}^{2}$ sur 9 par parcelle d'inventaire (Figure 2). Dans les différents lots, on a procédé à un inventaire systématique des espèces végétales présentes. Les paramètres spécifiques caractéristiques suivants ont été relevés :

- Identification de l'espèce,

- Dénombrement de pieds par espèce et pour toutes les espèces dans chaque lot inventorié,

- Evaluation du recouvrement aérien par espèce et par individu,

- Evaluation du recouvrement basal par espèce et par individu,

- sociabilité, type biologique et morphologique de chaque plante.

L'inventaire floristique de la strate herbacée était réalisé par la méthode phytosociologique sigmatiste de Braun Blanquet (1932) et toutes les espèces végétales contenues dans le lot inventorié de $1 \mathrm{~m}^{2}$ étaient comptées et 


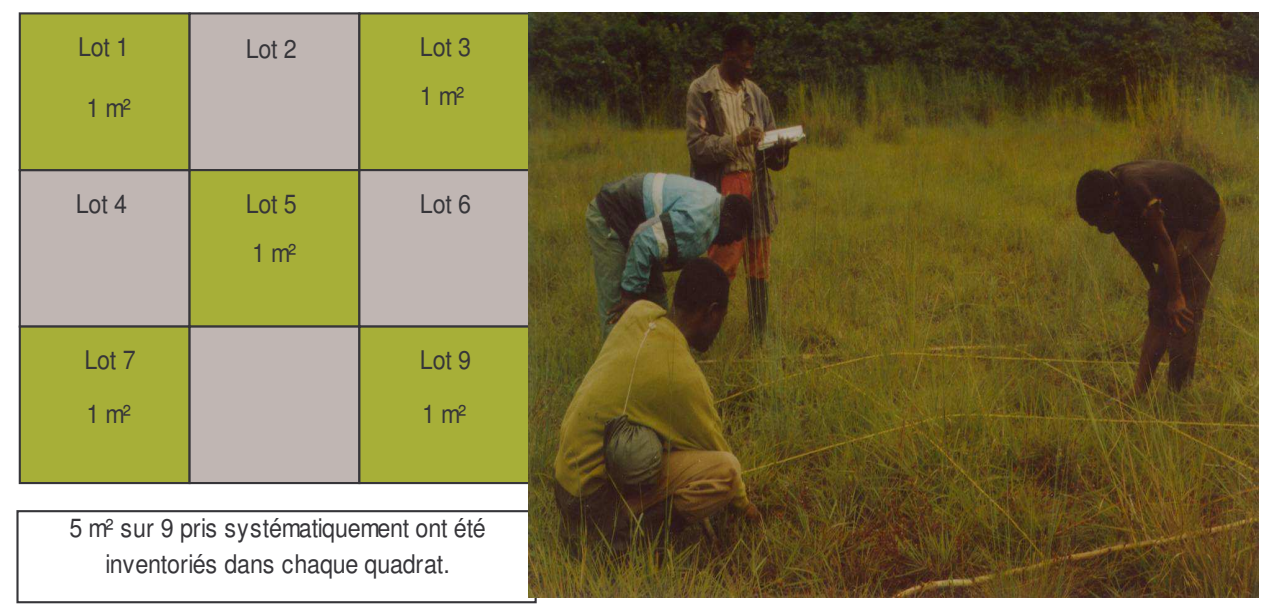

Figure 2: Dispositif de relevé de végétation.

enregistrées sur des fiches standard d'inventaire de la végétation. Dans toutes les placettes inventoriées pour estimer l'importance relative des espèces, l'échelle mixte d'abondance-dominance a été utilisée (Boudet, 1991),

$\mathrm{R}$ étant le recouvrement, l'échelle suivante a été utilisée :

+ : espèce présente à l'état d'individus isolés (abondance et recouvrement faibles) ;

1: espèce présente à l'état d'individus abondants, recouvrement faible $(\mathrm{R}<5 \mathrm{p}$. 100) ;

2: espèce présente à l'état d'individus abondants, recouvrement voisin de 5 p. 100 ;

3 : espèce dont le recouvrement peut atteindre le 1 / 3 du relevé ;

4 : espèce dont le recouvrement occupe entre 1 / 3 et $2 / 3$ du relevé $(\mathrm{R}=35$ à 65 p. 100)

5 : espèce dont le recouvrement occupe plus des 2 / 3 du relevé ( $\mathrm{R}>65$ p. 100).

Méthode d'analyse des données floristiques

Les données de cette étude ont été analysées avec l'outil EXCEL et le calcul des indices a été fait en utilisant les équations cidessous :

\section{Fréquence spécifique}

$$
F s=100 * \frac{f i}{N}
$$

Où :

Fs : Fréquence spécifique.

fi:Nombre de relevés dans lequel

l'espèce i est présente.

$\mathrm{N}$ : Nombre total de relevés.

Densité spécifique

$D s=\frac{n i}{N}$

Où :

Ds : Densité spécifique.

ni : Nombre d'individus de l'espèce $i$.

$\mathrm{N}$ : Nombre total de relevés.

$D s / h a=10.000 * D s$.

Densité relative de l'espèce $\mathrm{i}$

$D r=100 * \frac{n i}{N t}$

Où :

Dr : Densité relative de l'espèce i.

ni: Nombre d'individus de l'espèce i dans l'ensemble des relevés par clairière

Nt: Nombre total d'individus de toutes les espèces dans tous les relevés d'inventaire par clairière.

Dominance de l'espèce i

$D o=100 * \frac{f i}{\sum f i}$ 
Où :

Do : Dominance de l'espèce i.

fi: nombre de relevés d'inventaire dans lesquels l'espèce i apparaît

$\sum f i$ : Somme des nombres de relevés dans lesquelles toutes les espèces apparaissent. $\underline{\text { Abondance de l'espèce } \mathrm{i}}$

$$
A b s=\frac{n i}{f i}
$$

Où : Abs : Abondance de l'espèce i.

[(Ab*Fr)/D=100].

ni : Nombre d'individus de l'espèce i dans l'ensemble des relevés par clairière

L'équation (7) est l'équation de vérification de l'efficience de la collecte des données (Whilford, 1949).

\section{RESULTATS \\ Richesse floristique globale du milieu d'étude}

Le nombre d'espèces, de genres et de familles trouvés dans les clairières de Boumba-Bek est récapitulé dans le tableau 1.

L'inventaire floristique dans les six clairières d'étude a permis d'identifier au total 32 espèces ligneuses réparties en 29 genres appartenant à 20 familles et 68 espèces herbacées reparties en 53 genres et appartenant à 26 familles. L'identification des espèces herbacées permet ainsi de porter la liste des espèces du massif de Boumba-Bek en complément des études antérieures à 899 espèces appartenant à 137 familles. La composante floristique des ligneux bien que représentée par 32 espèces, est caractérisée par l'isolement des pieds observés çà et là dans les clairières et plus particulièrement, elle est rencontrée dans la galerie forestière qui traverse la clairière de Likolo. On n'a pas identifié d'espèces nouvelles différentes des espèces connues dans le massif, ce qui explique que l'on ne les ait pas inclus à nouveau dans la liste des espèces du milieu. Etant donné que les listes des espèces dressées par Nkongmeneck (1996) et Ekobo (1998) n'incluaient pas les herbacées, cette dernière composante floristique a été considérée comme une contribution de l'étude à l'amélioration de la connaissance sur la diversité floristique du milieu et aussi les a-ton inclus dans la liste nouvelle des espèces végétales du Parc National de Boumba-Bek.
La détermination du nombre d'espèces rencontrées par famille dans le cortège de la végétation des six clairières étudiées indique l'importance des différentes familles dans l'écosystème étudié. Des 26 familles d'herbacées identifiées comme colonisant le milieu, il est ressorti que la famille des Poaceae est la plus importante dans le cortège floristique du milieu d'étude. En effet, on a rencontré 18 espèces appartenant à cette famille sur un total de 68 , ce qui représente $26,5 \%$ d'espèces rencontrées.

Le peuplement graminoïde qui se compose de Poaceae et de Cyperaceae représente 39,7\% d'espèces rencontrées dans les relevés et 5 familles seulement sur les 26 existantes (Poaceae, Cyperaceae, Fabaceae, Commelianceae et Malastomataceae) représentent $60,3 \%$ des espèces des relevés. Il ressort du spectre biologique des clairières étudiées (Tableau 2) que le milieu est dominé par deux groupes de végétaux: le groupe composé des cinq familles (Poaceae, Cyperaceae, Fabaceae, Commelinaceae et Malastomataceae) qui compte $60,3 \%$ des espèces rencontrées et le reste, soit 21 familles et qui représente $39,7 \%$ des espèces rencontrées.

\section{Aire minimale phytosociologique}

La surface minimale d'inventaire significatif dans l'étude détermine le nombre de parcelles d'inventaire à partir duquel on n'enregistre plus l'apparition de nouvelles espèces. Les clairières de l'étude étant de superficies variables allant de 1,5 ha à plus de 200 ha, le nombre de parcelles d'inventaire était aussi variable proportionnellement. Ainsi, la superficie minimale d'étude quantitative des clairières forestières a été déterminée par accroissement progressif des superficies en fonction de l'apparition de nouvelles espèces, et la figure 3 présente l'écart entre l'aire minimale phytosociologique de l'étude et l'échantillonnage d'inventaire réalisé.

Il se dégage que les relevés d'inventaires de la végétation dans les six clairières d'étude étaient satisfaisants proportionnellement aux superficies des différentes clairières, permettant ainsi la validation de l'étude de la végétation ainsi réalisée. 
Tableau 1: Récapitulatif de la richesse spécifique des clairières d'étude.

\begin{tabular}{llccc}
\hline Milieu d'étude & $\begin{array}{l}\text { Composante } \\
\text { floristique }\end{array}$ & $\begin{array}{c}\text { Nombre } \\
\text { d'espèces }\end{array}$ & $\begin{array}{c}\text { Nombre de } \\
\text { genres }\end{array}$ & $\begin{array}{c}\text { Nombre de } \\
\text { familles }\end{array}$ \\
\hline Total pour toutes & Ligneux & $\mathbf{3 2}$ & $\mathbf{2 9}$ & $\mathbf{2 0}$ \\
les clairières & Herbacées & $\mathbf{6 8}$ & $\mathbf{5 3}$ & $\mathbf{2 6}$ \\
\hline Likolo & Ligneux & - & - & - \\
& Herbacées & 28 & 23 & 11 \\
\hline Batouka & Ligneux & - & - & - \\
& Herbacées & 21 & 18 & 9 \\
\hline Bako 1 & Ligneux & - & - & - \\
& Herbacées & 16 & 14 & 8 \\
\hline Bako 2 & Ligneux & - & - & - \\
& Herbacées & 17 & & 10 \\
\hline Sike 1 & Ligneux & - & - & - \\
& Herbacées & 19 & 16 & - \\
\hline Sike 2 & Ligneux & - & - & 9 \\
& Herbacées & 16 & 13 & \\
\hline
\end{tabular}

\section{Spectre biologique des familles dans l'aire d'étude}

Le décompte du nombre d'espèces rencontrées par famille a permis ainsi de déterminer le spectre biologique des familles dans les clairières sur schiste dans le massif forestier de Boumba-Bek (Tableau 2 et Figure 4). Il s'en dégage que le peuplement graminoïde représente pour l'ensemble des relevés $39,7 \%$ réparties en Poaceae $(26,5 \%)$ et Cyperaceae $(13,2 \%)$.

Les familles suivantes: Poaceae, Fabaceae, Commelinaceae et Melastomataceae qui représentent $47,1 \%$ des espèces identifiées sont représentées par 41 espèces au total et, selon les observations elles constituent les groupes d'espèces appétées par les herbivores. On constate donc que les familles les plus représentatives du cortège végétal des clairières sont aussi celles qui possèdent les espèces préférentiellement appétées par les herbivores sauvages (exception faite de la famille des Cyperaceae). Ceci confirme le rôle de pâturage naturel que ces clairières jouent dans le milieu et explique la concentration d'herbivores qu'on observe dans et autour de ces écosystèmes.

Le deuxième groupe important dans le milieu est constitué des familles suivantes: Balsaminaceae, Acanthaceae Euphorbiaceae, Malvaceae, Rubiaceae et Tiliaceae. Chaque famille de ce groupe est représentée par deux espèces et le groupe représente en importance, $17,4 \%$ dans le cortège végétal de la région.

Le troisième groupe est constitué de quinze familles, chacune étant représentée par une seule espèce. Au total, les quinze familles de ce groupe constituent $22,5 \%$ du cortège végétal des clairières d'étude.

L'analyse du spectre biologique amène à conclure que les clairières de cette étude sont des aires fourragères pour les herbivores sauvages. Ce rôle d'aire fourragère pour la faune sauvage détermine l'importance de ces écosystèmes pour l'aménagement des ressources animales de la forêt tropicale et notamment de la forêt du Bassin du Congo.

$\mathrm{La}$ famille des Cyperaceae est fortement représentée $(13,2 \%$ des espèces observées) ce qui, du fait de l'agression des espèces de cette famille sur la faune représente une menace importante pour les clairières forestières. En effet, on a observé que les clairières envahies par les Cyperaceae sont rapidement abandonnées par les animaux $\mathrm{du}$ fait des blessures faites à leurs robes et l'absence de leur fréquentation conduit à une régression de l'écosystème. Pour débarrasser les clairières forestières des Cyperaceae, il est préconisé des interventions d'enlèvement dans le cadre de l'aménagement des clairières dans le massif de Boumba-Bek. 
Tableau 2: Spectre biologique des différentes familles identifiées dans les clairières de Boumba-Bek.

\begin{tabular}{clcc}
\hline $\mathbf{N}^{\circ}$ & Famille & $\begin{array}{c}\text { Nombre } \\
\text { d'espèces }\end{array}$ & $\begin{array}{c}\text { Pourcentage } \\
\text { dans les relevés }\end{array}$ \\
\hline 1 & Poaceae & 18 & 26,47 \\
2 & Cyperaceae & 9 & 13,24 \\
3 & Fabaceae & 7 & 10,29 \\
4 & Commelinaceae & 4 & 5,88 \\
5 & Melastomataceae & 3 & 4,41 \\
6 & Balsaminaceae & 2 & 2,94 \\
7 & Acanthaceae & 2 & 2,94 \\
8 & Euphorbiaceae & 2 & 2,94 \\
9 & Malvaceae & 2 & 2,94 \\
10 & Rubiaceae & 2 & 2,94 \\
11 & Tiliaceae & 2 & 2,94 \\
12 & Araceae & 1 & 1,47 \\
13 & Araliaceae & 1 & 1,47 \\
14 & Cesalpiniaceae & 1 & 1,47 \\
15 & Capparaceae & 1 & 1,47 \\
16 & Cucurbitaceae & 1 & 1,47 \\
17 & Convolvulaceae & 1 & 1,47 \\
18 & Dowalliaceae & 1 & 1,47 \\
19 & Nympheaceae & 1 & 1,47 \\
20 & Lamiaceae & 1 & 1,47 \\
21 & Liliaceae & 1 & 1,47 \\
22 & Onagraceae & 1 & 1,47 \\
23 & Polygonaceae & 1 & 1,47 \\
24 & Scrophulariaceae & 1 & 1,47 \\
25 & thelypteridaceae & 1 & 1,47 \\
26 & Apiaceae & 1 & 1,47 \\
\hline & Total & $\mathbf{6 8}$ & $\mathbf{1 0 0 , 0 0}$ \\
\hline
\end{tabular}

Composition floristique et diversité spécifique

Caractérisation par la Dominance, l'Abondance et la Fréquence des espèces

Dans l'ensemble des relevés de la végétation, 68 espèces ont été rencontrées dans les clairières étudiées. Classées par rapport au référentiel de dominance décroissante à Likolo, les valeurs de dominance, d'abondance spécifique et de fréquence pour les espèces rencontrées dans les clairières ont été obtenues par calcul en utilisant les formules énoncées dans la méthodologie. Les tableaux 3 à 8 récapitulent les résultats obtenus pour chacune des six clairières et la relation de Wilford, [(Abondance spécifique) $x$ (Fréquence spécifique)] / Densité spécifique $=100$
(Greig-Smith, 1983), est vérifiée pour chacune des données.

Cette relation permet la vérification des calculs sur les paramètres de la végétation. Cette relation s'est avérée vérifiée pour toutes les données de cette étude, confirmant l'efficacité des calculs.

Dominance spécifique: La caractérisation globale des espèces par leur dominance est représentée par la figure 5. Il en ressort que parmi les espèces végétales rencontrées dans les clairières étudiées, cinq à savoir : Loudetia simplex, Cyanotis lanata, Striga aspera, Crotalaria lachnophora, Triumfetta pentandra, Eragrostis atrovirens sont rencontrées dans toutes les clairières. $L$. simplex affiche la plus grande valeur de 

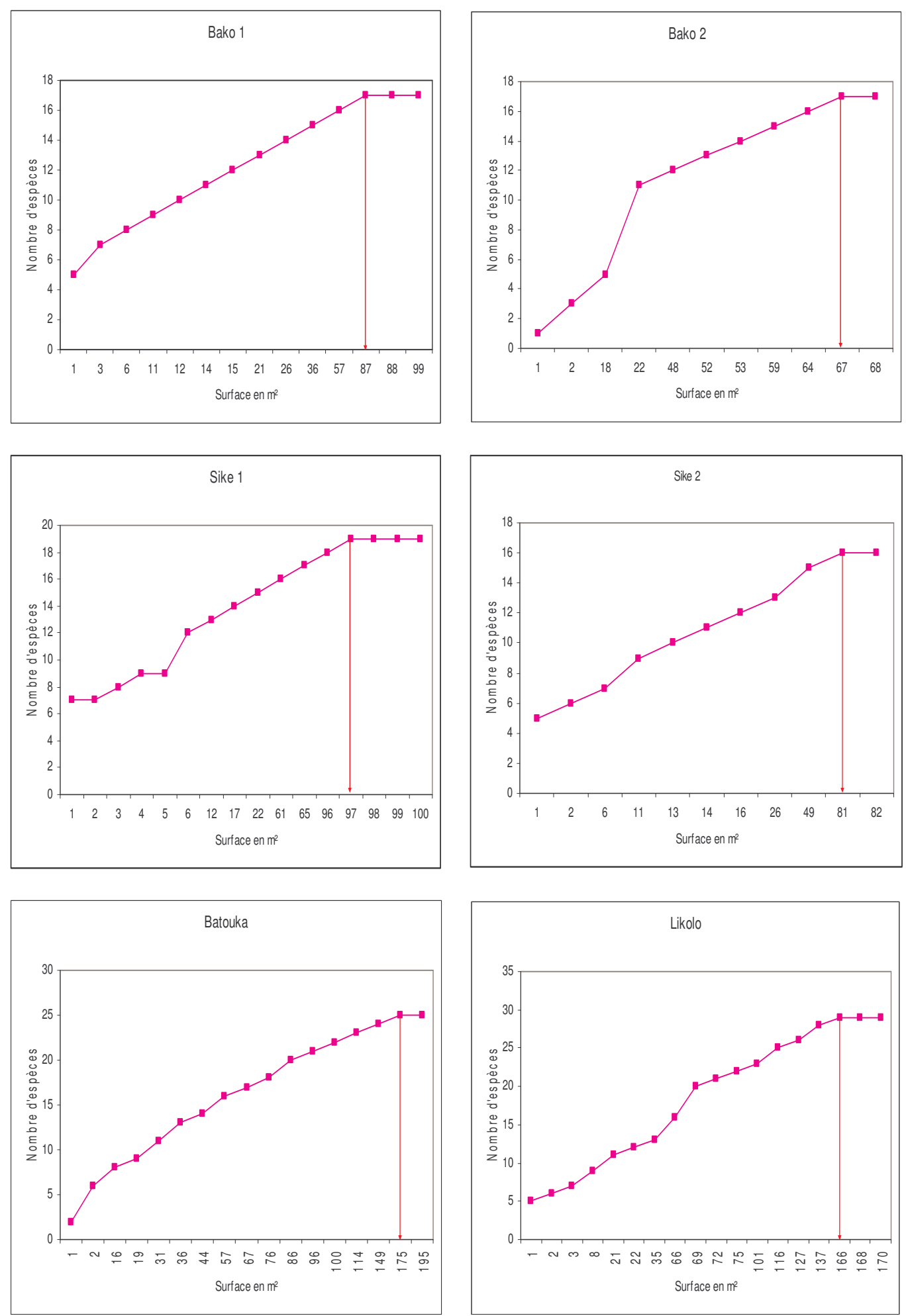

Figure 3: Détermination de l'aire minimale phytosociologique dans les clairières d'étude. 

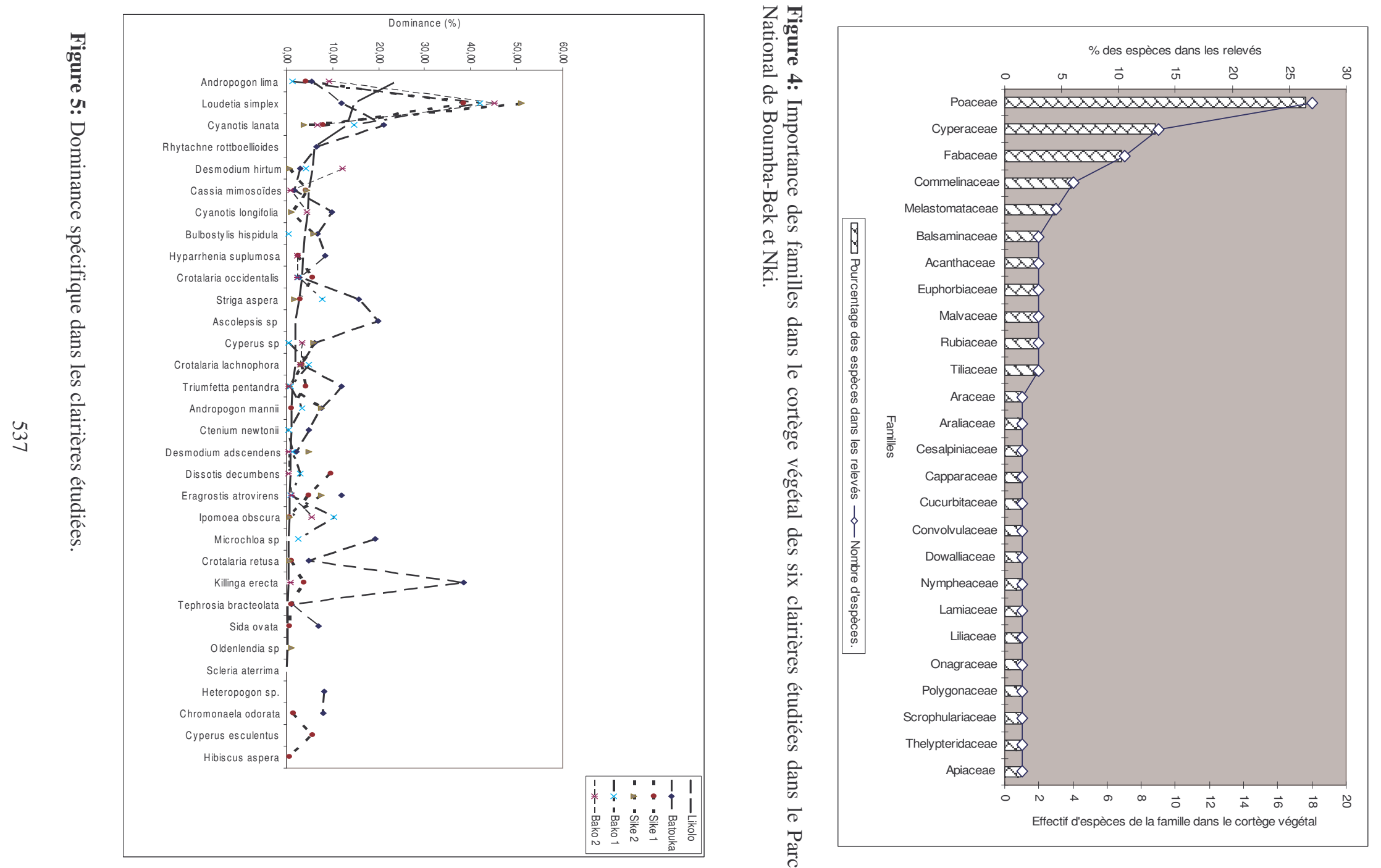
Tableau 3: Récapitulatif des valeurs des paramètres spécifiques de la végétation à Likolo.

\begin{tabular}{|c|c|c|c|c|c|c|}
\hline \multicolumn{7}{|c|}{$\mathrm{N}=395$} \\
\hline Espèces & $\begin{array}{l}\text { NIE } \\
\text { (ni) }\end{array}$ & $\begin{array}{c}\text { NEE } \\
\text { (fi) }\end{array}$ & $\begin{array}{c}\text { Domi } \\
(\%)\end{array}$ & $\mathbf{A S}$ & $\begin{array}{c}\text { FS } \\
(\%)\end{array}$ & DS \\
\hline Andropogon lima & 3794 & 304 & 23,84 & 12,48 & 76,96 & 9,61 \\
\hline Loudetia simplex & 1558 & 187 & 14,67 & 8,33 & 47,34 & 3,94 \\
\hline Cyanotis lanata & 5358 & 169 & 13,25 & 31,70 & 42,78 & 13,56 \\
\hline Rhytachne rottboellioides & 512 & 77 & 6,04 & 6,65 & 19,49 & 1,30 \\
\hline Desmodium hirtum & 212 & 73 & 5,73 & 2,90 & 18,48 & 0,54 \\
\hline Cassia mimosoüdes & 99 & 62 & 4,86 & 1,60 & 15,70 & 0,25 \\
\hline Cyanotis longifolia & 468 & 59 & 4,63 & 7,93 & 14,94 & 1,18 \\
\hline Bulbostylis hispidula & 193 & 50 & 3,92 & 3,86 & 12,66 & 0,49 \\
\hline Hyparrhenia suplumosa & 301 & 44 & 3,45 & 6,84 & 11,14 & 0,76 \\
\hline Crotalaria occidentalis & 216 & 42 & 3,29 & 5,14 & 10,63 & 0,55 \\
\hline striga aspera & 666 & 35 & 2,75 & 19,03 & 8,86 & 1,69 \\
\hline Ascolepsis sp & 210 & 25 & 1,96 & 8,40 & 6,33 & 0,53 \\
\hline Cyperus sp & 142 & 25 & 1,96 & 5,68 & 6,33 & 0,36 \\
\hline Crotalaria lachnophora & 64 & 24 & 1,88 & 2,67 & 6,08 & 0,16 \\
\hline Triumfetta pentandra & 34 & 17 & 1,33 & 2,00 & 4,30 & 0,09 \\
\hline Andropogon mannii & 42 & 12 & 0,94 & 3,50 & 3,04 & 0,11 \\
\hline Ctenium newtonii & 101 & 12 & 0,94 & 8,42 & 3,04 & 0,26 \\
\hline Desmodium adscendens & 31 & 11 & 0,86 & 2,82 & 2,78 & 0,08 \\
\hline Dissotis decumbens & 25 & 11 & 0,86 & 2,27 & 2,78 & 0,06 \\
\hline Eragrostis atrovirens & 31 & 8 & 0,63 & 3,88 & 2,03 & 0,08 \\
\hline Ipomoea obscura & 12 & 7 & 0,55 & 1,71 & 1,77 & 0,03 \\
\hline Microchloa sp & 182 & 5 & 0,39 & 36,40 & 1,27 & 0,46 \\
\hline Crotalaria retusa & 8 & 4 & 0,31 & 2,00 & 1,01 & 0,02 \\
\hline Killing a erecta & 7 & 4 & 0,31 & 1,75 & 1,01 & 0,02 \\
\hline Tephrosia bracteolata & 3 & 3 & 0,24 & 1,00 & 0,76 & 0,01 \\
\hline Sida ovata & 9 & 2 & 0,16 & 4,50 & 0,51 & 0,02 \\
\hline Oldenlendia $s p$ & 4 & 2 & 0,16 & 2,00 & 0,51 & 0,01 \\
\hline \multirow[t]{2}{*}{ Scleria aterrima } & 1 & 1 & 0,08 & 1,00 & 0,25 & 0,00 \\
\hline & 14283 & 1275 & 100,00 & & & \\
\hline
\end{tabular}

dominance de tous les relevés dans la clairière de Sike 2 avec 51,08\%. La plus faible valeur de dominance affichée par cette espèce est de $11,84 \%$ à Batouka, suivi de Likolo avec $14,67 \%$. Partout ailleurs, L. simplex a obtenu des valeurs de dominance assez élevée respectivement de $38,29 \%$ à Sike1, $42,04 \%$ à Bako1, 45,05\% à Bako2 et qui culmine à Sike2 avec 51,08\%. A l'exception de Kilinga erecta (Dominance de 38,40\% à Batouka), toutes les autres espèces dans toutes les clairières ont affiché des valeurs de dominance inférieure à $25 \%$, le maximal étant 23,84\% affiché par Andropogon lima à Likolo. Ainsi, du point de vue de la dominance, L. simplex influence la physionomie de toutes les clairières étudiées dans le massif de Boumba-Bek.

Abondance spécifique: L'abondance spécifique exprime la moyenne du nombre d'individus d'une espèce donnée par rapport à l'ensemble des relevés dans lesquelles cette espèce apparaît. La figure 6 est la représentation de l'abondance spécifique 
Tableau 4: Récapitulatif des valeurs des paramètres spécifiques de la végétation à Batouka.

\begin{tabular}{|c|c|c|c|c|c|c|}
\hline & $\mathrm{N}=2$ & & & & & \\
\hline Espèce & $\begin{array}{c}\text { NIE } \\
\text { (ni) }\end{array}$ & $\begin{array}{c}\text { NEE } \\
\text { (fi) }\end{array}$ & $\begin{array}{c}\text { Domi. } \\
(\%)\end{array}$ & $\mathbf{A S}$ & $\begin{array}{c}\text { FS } \\
(\%)\end{array}$ & DS \\
\hline Loudetia simplex & 1942 & 164 & 25,91 & 11,84 & 80,00 & 9,47 \\
\hline Andropogon lima & 334 & 62 & 9,79 & 5,39 & 30,24 & 1,63 \\
\hline Cyanotis longifolia & 584 & 60 & 9,48 & 9,73 & 29,27 & 2,85 \\
\hline Striga aspera & 930 & 59 & 9,32 & 15,76 & 28,78 & 4,54 \\
\hline Cyanotis sp & 1093 & 52 & 8,21 & 21,02 & 25,37 & 5,33 \\
\hline Crotalaria lachnophora & 111 & 35 & 5,53 & 3,17 & 17,07 & 0,54 \\
\hline Hyparrhenia subplumosa & 207 & 25 & 3,95 & 8,28 & 12,20 & 1,01 \\
\hline Ascolepsis sp. & 439 & 22 & 3,48 & 19,95 & 10,73 & 2,14 \\
\hline Desmodium hirtum & 62 & 21 & 3,32 & 2,95 & 10,24 & 0,30 \\
\hline Rhytachne rottboellioides & 123 & 19 & 3,00 & 6,47 & 9,27 & 0,60 \\
\hline Cassia mimosö̈des & 22 & 14 & 2,21 & 1,57 & 6,83 & 0,11 \\
\hline Eragraotis atrovirens & 166 & 14 & 2,21 & 11,86 & 6,83 & 0,81 \\
\hline Crotalaria retusa & 66 & 14 & 2,21 & 4,71 & 6,83 & 0,32 \\
\hline Heteropogon $s p$. & 105 & 13 & 2,05 & 8,08 & 6,34 & 0,51 \\
\hline Andropogon manii & 76 & 10 & 1,58 & 7,60 & 4,88 & 0,37 \\
\hline Ctenium newtonii & 49 & 10 & 1,58 & 4,90 & 4,88 & 0,24 \\
\hline Cyperus sp & 48 & 8 & 1,26 & 6,00 & 3,90 & 0,23 \\
\hline Bulbostilis hispidula & 53 & 8 & 1,26 & 6,63 & 3,90 & 0,26 \\
\hline Sida ovata & 34 & 5 & 0,79 & 6,80 & 2,44 & 0,17 \\
\hline Killing a erecta & 192 & 5 & 0,79 & 38,40 & 2,44 & 0,94 \\
\hline Crotalaria occidentalis & 8 & 3 & 0,47 & 2,67 & 1,46 & 0,04 \\
\hline Microchloa sp. & 58 & 3 & 0,47 & 19,33 & 1,46 & 0,28 \\
\hline Desmodium adscendens & 6 & 3 & 0,47 & 2,00 & 1,46 & 0,03 \\
\hline Triumffeta pentendra & 24 & 2 & 0,32 & 12,00 & 0,98 & 0,12 \\
\hline Tephrosia bracteolata & 1 & 1 & 0,16 & 1,00 & 0,49 & 0,00 \\
\hline \multirow[t]{2}{*}{ Chromolaena odorata } & 8 & 1 & 0,16 & 8,00 & 0,49 & 0,04 \\
\hline & 6741 & 633 & 100,00 & & & \\
\hline
\end{tabular}

obtenue à l'issue des inventaires dans les six clairières d'étude. Il s'en dégage qu'à l'exception de L. simplex à Likolo $(28,81)$, de Cyanotis lanata respectivement dans les clairières de Sike2 $(45,57)$, Likolo $(31,70)$, Bako1 (26,83) et Bako2 (22,06), de Striga aspera à Bako1 (42,58), et de Microchloa sp à Likolo $(36,40)$, le reste des espèces du cortège floristique des clairières étudiées affichent des valeurs d'abondance spécifique inférieures à 20 individus. Les clairières d'études sont ainsi caractérisées par une abondance spécifique comprise entre 0 et 50 individus avec une moyenne inférieure à 20 individus par unité de comptage.
Fréquence spécifique: La fréquence représente le pourcentage d'apparition d'une espèce dans l'ensemble des échantillons du relevé. La figure 7 donne une représentation graphique des valeurs de fréquence spécifique obtenues de cette étude. Il ressort de cette étude qu'à l'exception de Andropogon lima à Likolo (fréquence égale 76,96\%), de cyanotis lanata à likolo et de L. simplex dans les six clairières, l'ensemble des espèces du cortège végétal ont présenté des valeurs de fréquence au plus égale à $30 \%$. Du point de vue de la fréquence, $L$. simplex est l'espèce végétale la plus fréquemment rencontrée des clairières 

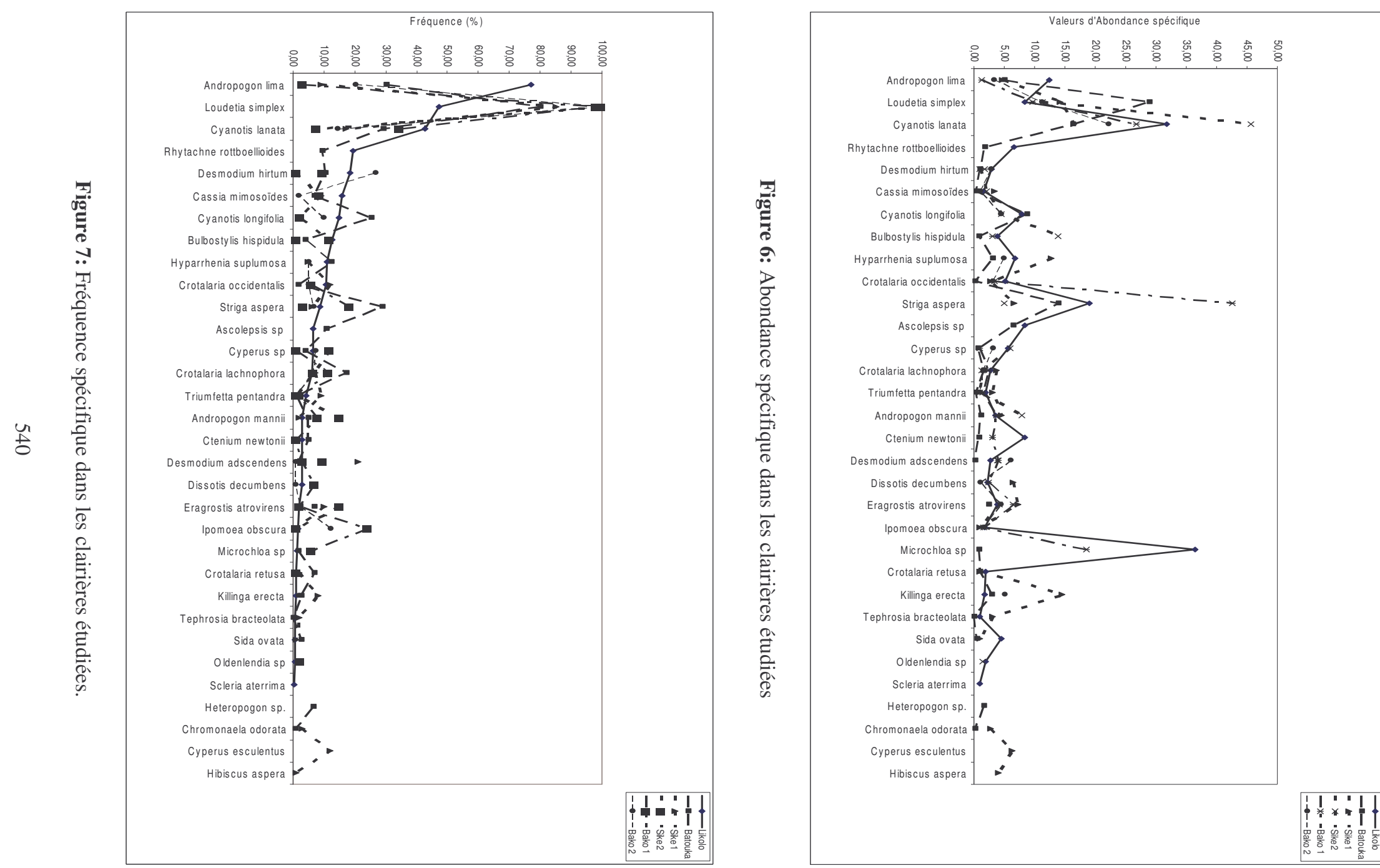
Tableau 5: Récapitulatif des valeurs des paramètres spécifiques de la végétation à Sike 1.

\begin{tabular}{|c|c|c|c|c|c|c|}
\hline & & $\mathrm{N}=10$ & & & & \\
\hline Espèce & $\begin{array}{l}\text { NIE } \\
\text { (ni) }\end{array}$ & $\begin{array}{c}\text { NEE } \\
\text { (fi) }\end{array}$ & $\begin{array}{c}\text { Domi. } \\
(\%)\end{array}$ & AS & $\begin{array}{c}\text { FS } \\
(\%) \\
\end{array}$ & DS \\
\hline Loudetia simplex & 1200 & 85 & 38,29 & 14,12 & 85 & 12 \\
\hline Desmodium adscendens & 134 & 21 & 9,46 & 6,38 & 21 & 1,34 \\
\hline Cyanotis lanata & 279 & 17 & 7,66 & 16,41 & 17 & 2,79 \\
\hline Cyperus esculentus & 76 & 12 & 5,41 & 6,33 & 12 & 0,76 \\
\hline Crotalaria occidentalis & 32 & 12 & 5,41 & 2,67 & 12 & 0,32 \\
\hline Eragrostis atrovirens & 72 & 10 & 4,50 & 7,20 & 10 & 0,72 \\
\hline Andropogon lima & 42 & 9 & 4,05 & 4,67 & 9 & 0,42 \\
\hline Cassia mimosoïdes & 31 & 9 & 4,05 & 3,44 & 9 & 0,31 \\
\hline Triumfetta pentandra & 27 & 9 & 4,05 & 3,00 & 9 & 0,27 \\
\hline Killinga erecta & 116 & 8 & 3,60 & 14,50 & 8 & 1,16 \\
\hline Crotalaria lachnopholia & 26 & 7 & 3,15 & 3,71 & 7 & 0,26 \\
\hline Striga aspera & 40 & 6 & 2,70 & 6,67 & 6 & 0,4 \\
\hline Hiparrhenia subplumosa & 64 & 5 & 2,25 & 12,80 & 5 & 0,64 \\
\hline Chromonalae odorata & 8 & 3 & 1,35 & 2,67 & 3 & 0,08 \\
\hline Crotalaria retusa & 2 & 2 & 0,90 & 1,00 & 2 & 0,02 \\
\hline Tephrosia bracteolata & 6 & 2 & 0,90 & 3,00 & 2 & 0,06 \\
\hline Andropogon manii & 9 & 2 & 0,90 & 4,50 & 2 & 0,09 \\
\hline Ipomoea obscura & 1 & 1 & 0,45 & 1,00 & 1 & 0,01 \\
\hline Hibiscus aspera & 4 & 1 & 0,45 & 4,00 & 1 & 0,04 \\
\hline \multirow[t]{2}{*}{ Sida ovata } & 1 & 1 & 0,45 & 1,00 & 1 & 0,01 \\
\hline & 2170 & 222 & 100,00 & & & \\
\hline
\end{tabular}

$\mathrm{AS}=$ Abondance spécifique $; \mathrm{FS}=$ Fréquence spécifique $; \mathrm{DS}=$ Densité spécifique

d'étude, avec les fréquences de $100 \%$ (à Sike 2), 98,40\% (à Bako 2), 98,10\% (à Bako 1), $85 \%$ (à Sike 1), $80 \%$ (à Batouka) et $47,34 \%$ (à Likolo).

\section{Caractérisation de la diversité biologique}

L'analyse de la distribution des espèces a permis de caractériser les écosystèmes étudiés en termes de diversité de leurs composants biologiques. Les figures 8,9 et 10 , obtenues pour chaque clairière en représentant dans un même graphique et pour chaque quadra étudié, le nombre d'individus inventoriés toutes espèces végétales confondues et le nombre d'espèces végétales rencontrées dans ledit quadra permettent une caractérisation de la diversité biologique du milieu étudié. Le diagramme obtenu traduit la physionomie de la diversité quantitative (nombre d'individus rencontrés) et qualitative (nombre d'espèces rencontrées) dans le quadra ou lot d'inventaire. Etendu à l'ensemble des relevés d'inventaire, le diagramme de distribution de présence/fréquence rend compte de la richesse quantitative et qualitative du milieu étudié.

Lorsque l'histogramme du nombre d'individus comptés dans un quadra est au dessus de la courbe correspondante du nombre d'espèces dans ledit quadra, le milieu est plus riche (nombre d'individus) que diversifié (nombre d'espèces). Par contre, si l'histogramme du nombre d'individus comptés par quadra est en dessous de la courbe du nombre d'espèces inventoriées dans le quadra, le milieu est plus diversifié (nombre d'espèces rencontrées) que riche (nombre d'individus). La richesse du milieu en individus est un paramètre important dans la biomasse végétale des clairières alors que sa diversité spécifique est un paramètre important dans la qualité du pâturage. 
Tableau 6: Récapitulatif des valeurs des paramètres spécifiques de la végétation à Sike 2.

\begin{tabular}{|c|c|c|c|c|c|c|}
\hline \multicolumn{7}{|c|}{$\mathrm{N}=95$} \\
\hline Espèces & $\begin{array}{l}\text { NIE } \\
\text { (ni) }\end{array}$ & $\begin{array}{c}\text { NEE } \\
\text { (fi) }\end{array}$ & $\begin{array}{c}\text { Domi. } \\
(\%)\end{array}$ & $\mathbf{A S}$ & $\begin{array}{l}\text { FS } \\
(\%)\end{array}$ & DS \\
\hline Loudetia simplex & 1065 & 95 & 51,08 & 11,21 & 100 & 11,21 \\
\hline Eragrostis atrovirens & 58 & 14 & 7,53 & 4,14 & 14,74 & 0,61 \\
\hline Andropogon manii & 110 & 14 & 7,53 & 7,86 & 14,74 & 1,16 \\
\hline bulbostylis hispidula & 152 & 11 & 5,91 & 13,82 & 11,58 & 1,60 \\
\hline Cyperus sp & 66 & 11 & 5,91 & 6,00 & 11,58 & 0,69 \\
\hline Desmodium adscendens & 35 & 9 & 4,84 & 3,89 & 9,47 & 0,37 \\
\hline Cassia mimosoïdes & 17 & 8 & 4,30 & 2,13 & 8,42 & 0,18 \\
\hline Cyanotis $S p$ & 319 & 7 & 3,76 & 45,57 & 7,37 & 3,36 \\
\hline Crotalaria lachnophora & 8 & 6 & 3,23 & 1,33 & 6,32 & 0,08 \\
\hline Striga sp & 15 & 3 & 1,61 & 5,00 & 3,16 & 0,16 \\
\hline Cyanotis longifolia & 9 & 2 & 1,08 & 4,50 & 2,11 & 0,09 \\
\hline Oldenlandia sp & 3 & 2 & 1,08 & 1,50 & 2,11 & 0,03 \\
\hline Triumfetta pendra & 1 & 1 & 0,54 & 1,00 & 1,05 & 0,01 \\
\hline Ipomoea obscura & 1 & 1 & 0,54 & 1,00 & 1,05 & 0,01 \\
\hline Desmodium hirtum & 1 & 1 & 0,54 & 1,00 & 1,05 & 0,01 \\
\hline \multirow[t]{2}{*}{ crotalaria retusa } & 1 & 1 & 0,54 & 1,00 & 1,05 & 0,01 \\
\hline & 1861 & 186 & 100,00 & & & \\
\hline
\end{tabular}

NIE = Nombre d'individus de l'espèce ; NEE = Nombre d'échantillon de l'espèce; Domi. = Dominance,

$\mathrm{AS}=$ Abondance spécifique $; \mathrm{FS}=$ Fréquence spécifique $; \mathrm{DS}=$ Densité spécifique

Tableau 7: Récapitulatif des valeurs des paramètres spécifiques de la végétation à Bako 1 .

\begin{tabular}{|c|c|c|c|c|c|c|}
\hline \multicolumn{7}{|c|}{$\mathrm{N}=110$} \\
\hline Espèce & $\begin{array}{c}\text { NIE } \\
\text { (ni) }\end{array}$ & $\begin{array}{c}\text { NEE } \\
\text { (fi) }\end{array}$ & $\begin{array}{c}\text { Domi. } \\
(\%)\end{array}$ & $\mathbf{A S}$ & $\begin{array}{c}\text { FS } \\
(\%)\end{array}$ & DS \\
\hline Loudetia simplex & 997 & 103 & 42,04 & 9,68 & 98,10 & 9,50 \\
\hline Cyanotis sp & 966 & 36 & 14,69 & 26,83 & 34,29 & 9,20 \\
\hline Ipomoea obscura & 42 & 25 & 10,20 & 1,68 & 23,81 & 0,40 \\
\hline Striga $s p$ & 809 & 19 & 7,76 & 42,58 & 18,10 & 7,70 \\
\hline Crotalaria lachnophora & 30 & 12 & 4,90 & 2,50 & 11,43 & 0,29 \\
\hline Desmodium hirtum & 17 & 10 & 4,08 & 1,70 & 9,52 & 0,16 \\
\hline Andropogon manii & 30 & 8 & 3,27 & 3,75 & 7,62 & 0,29 \\
\hline Dissotis decumbens & 17 & 7 & 2,86 & 2,43 & 6,67 & 0,16 \\
\hline Microchloa sp & 111 & 6 & 2,45 & 18,50 & 5,71 & 1,06 \\
\hline Crotalaria occidentalis & 20 & 6 & 2,45 & 3,33 & 5,71 & 0,19 \\
\hline Andropogon lima & 4 & 3 & 1,22 & 1,33 & 2,86 & 0,04 \\
\hline Desmodium adscendens & 12 & 3 & 1,22 & 4,00 & 2,86 & 0,11 \\
\hline Eragrostis atrovirens & 13 & 2 & 0,82 & 6,50 & 1,90 & 0,12 \\
\hline Triumfetta pentandra & 3 & 2 & 0,82 & 1,50 & 1,90 & 0,03 \\
\hline Bulbostylis hipidula & 3 & 1 & 0,41 & 3,00 & 0,95 & 0,03 \\
\hline Cyperus sp & 1 & 1 & 0,41 & 1,00 & 0,95 & 0,01 \\
\hline \multirow[t]{2}{*}{ Ctenium newtonii } & 3 & 1 & 0,41 & 3,00 & 0,95 & 0,03 \\
\hline & 3078 & 245 & 100,00 & & & \\
\hline
\end{tabular}

NIE = Nombre d'individus de l'espèce ; NEE = Nombre d'échantillon de l'espèce; Domi. = Dominance,

$\mathrm{AS}=$ Abondance spécifique $; \mathrm{FS}=$ Fréquence spécifique $; \mathrm{DS}=$ Densité spécifique 
Tableau 8: Récapitulatif des valeurs des paramètres spécifiques de la végétation à Bako 2.

\begin{tabular}{|c|c|c|c|c|c|c|}
\hline \multicolumn{7}{|c|}{$\mathrm{N}=125$} \\
\hline Espèces & $\begin{array}{l}\text { NIE } \\
\text { (ni) }\end{array}$ & $\begin{array}{c}\text { NEE } \\
\text { (fi) }\end{array}$ & $\begin{array}{c}\text { Domi. } \\
(\%)\end{array}$ & AS & $\begin{array}{c}\text { FS } \\
(\%)\end{array}$ & DS \\
\hline Loudetia simplex & 1367 & 123 & 45,05 & 11,11 & 98,4 & 10,936 \\
\hline Desmodium hirtum & 91 & 33 & 12,09 & 2,76 & 26,4 & 0,728 \\
\hline Andropogon lima & 80 & 25 & 9,16 & 3,20 & 20 & 0,64 \\
\hline Cyanotis sp & 397 & 18 & 6,59 & 22,06 & 14,4 & 3,176 \\
\hline Ipomoea obscura & 24 & 15 & 5,49 & 1,60 & 12 & 0,192 \\
\hline Cyanotis longifolia & 53 & 12 & 4,40 & 4,42 & 9,6 & 0,424 \\
\hline Cyperus sp & 27 & 9 & 3,30 & 3,00 & 7,2 & 0,216 \\
\hline Striga sp & 895 & 8 & 2,93 & 111,88 & 6,4 & 7,16 \\
\hline Crotalaria lachnophora & 12 & 8 & 2,93 & 1,50 & 6,4 & 0,096 \\
\hline Crotalaria occidentalis & 18 & 6 & 2,20 & 3,00 & 4,8 & 0,144 \\
\hline Hyparrhenia subplumosa & 29 & 6 & 2,20 & 4,83 & 4,8 & 0,232 \\
\hline Eragrostis atrovirens & 13 & 3 & 1,10 & 4,33 & 2,4 & 0,104 \\
\hline Cassia mimosö̈des & 2 & 2 & 0,73 & 1,00 & 1,6 & 0,016 \\
\hline Killing a erecta & 10 & 2 & 0,73 & 5,00 & 1,6 & 0,08 \\
\hline Triumfetta pentandra & 1 & 1 & 0,37 & 1,00 & 0,8 & 0,008 \\
\hline Desmodium adscendens & 6 & 1 & 0,37 & 6,00 & 0,8 & 0,048 \\
\hline \multirow[t]{2}{*}{ Dissotis decubens } & 1 & 1 & 0,37 & 1,00 & 0,8 & 0,008 \\
\hline & 3026 & 273 & 100,00 & & & \\
\hline
\end{tabular}

NIE = Nombre d'individus de l'espèce ; NEE = Nombre d'échantillon de l'espèce; Domi. = Dominance, $\mathrm{AS}=$ Abondance spécifique $; \mathrm{FS}=$ Fréquence spécifique $; \mathrm{DS}=$ Densité spécifique

Tableau 9: Caractérisation des clairières.

\begin{tabular}{lccccc}
\hline Clairière & $\begin{array}{c}\text { Superficie } \\
\text { inventoriée }\left(\mathbf{e n ~}^{\mathbf{2}} \mathbf{)}\right. \\
\text { = Nombre de quadra }\end{array}$ & $\begin{array}{c}\text { HNI }>\text { CNE } \\
(=\text { plus riche que } \\
\text { diversifié) }\end{array}$ & $\begin{array}{c}\text { \% de placettes } \\
\text { riches }\end{array}$ & $\begin{array}{c}\text { HNI < CNE } \\
\text { (= plus diversifié que } \\
\text { riche) }\end{array}$ & $\begin{array}{c}\text { \% de placettes } \\
\text { diversifiées }\end{array}$ \\
\hline Bako 1 & 110 & 5 & 5 & 105 & 95 \\
Bako 2 & 125 & 3 & 2 & 122 & 98 \\
Sike 1 & 100 & 31 & 31 & 69 & 69 \\
Sike 2 & 95 & 12 & 13 & 83 & 87 \\
Batouka & 205 & 37 & 18 & 168 & 82 \\
Likolo & 395 & 18 & 5 & 377 & 95 \\
\hline
\end{tabular}

$\mathrm{HNI}=$ Histogramme du nombre d'individus ; CNE = Courbe du nombre d'espèces

Le tableau 9 ci-dessus donne les caractéristiques des clairières en termes de nombre de placettes riches et/ou diversifiées. Il s'en dégage que les clairières sur schiste du massif de Boumba Bek sont plus diversifiées que riches. En effet, le pourcentage de placettes riches dans l'échantillonnage d'étude varie de $2 \%$ à Bako 2 à $31 \%$ à Sike 1 . A l'inverse, le pourcentage des placettes diversifiées varie de $69 \%$ à Sike 1 à $98 \%$ à Bako 1, ce qui permet d'affirmer que la végétation herbacée dans les clairières sur schiste du massif de Boumba Bek est plus diversifiée que riche. Ainsi, l'apparente homogénéité de la physionomie des clairières marquée parfois par la domination d'une seule espèce cache la réalité de la grande diversité floristique de ces écosystèmes.

La caractérisation de la diversité biologique qualitative et quantitative a ainsi permis de mettre en évidence les ressemblances physionomiques générales entre les clairières étudiées et les différences dans les unités d'échantillonnage à l'échelle des placettes et sous placettes. 
(A)

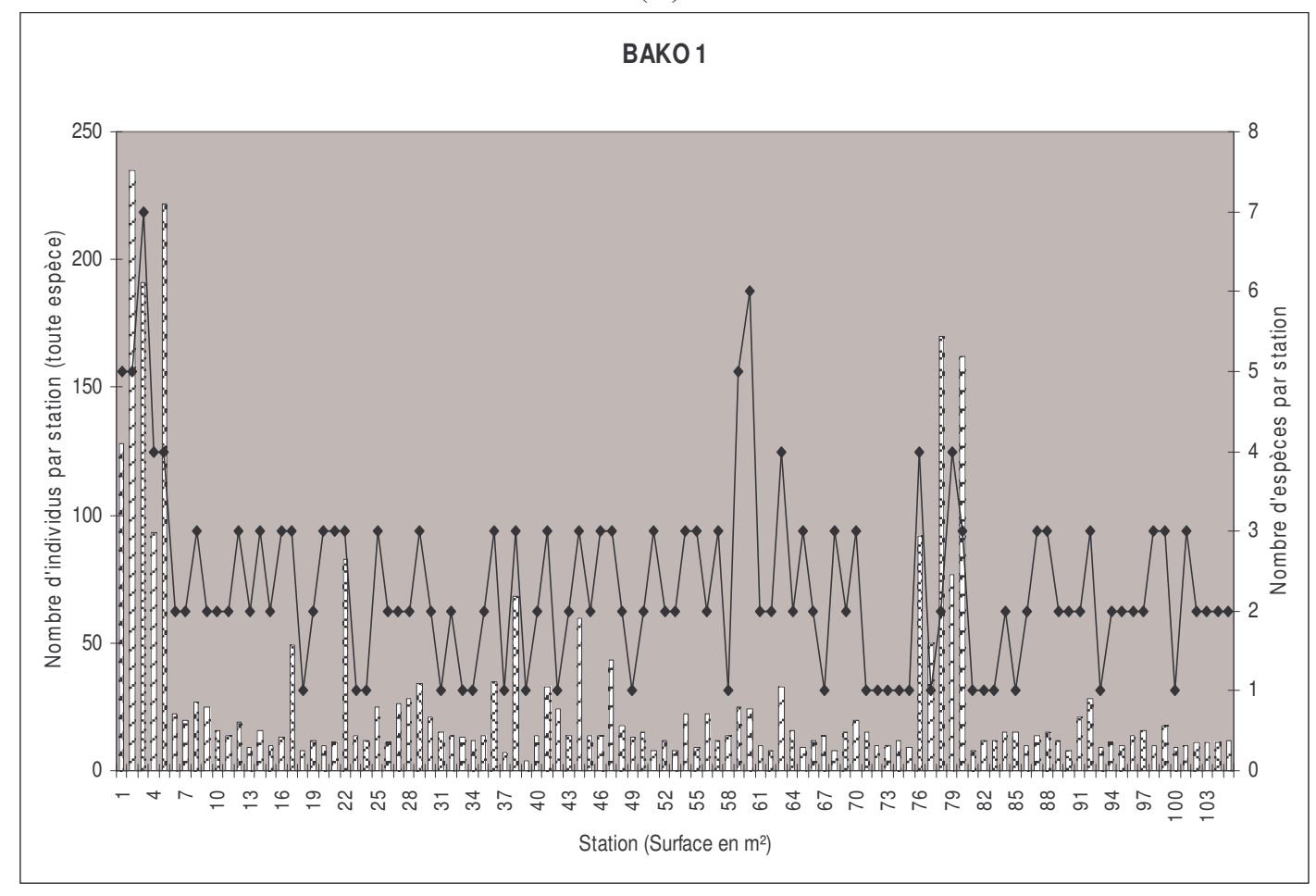

(B)

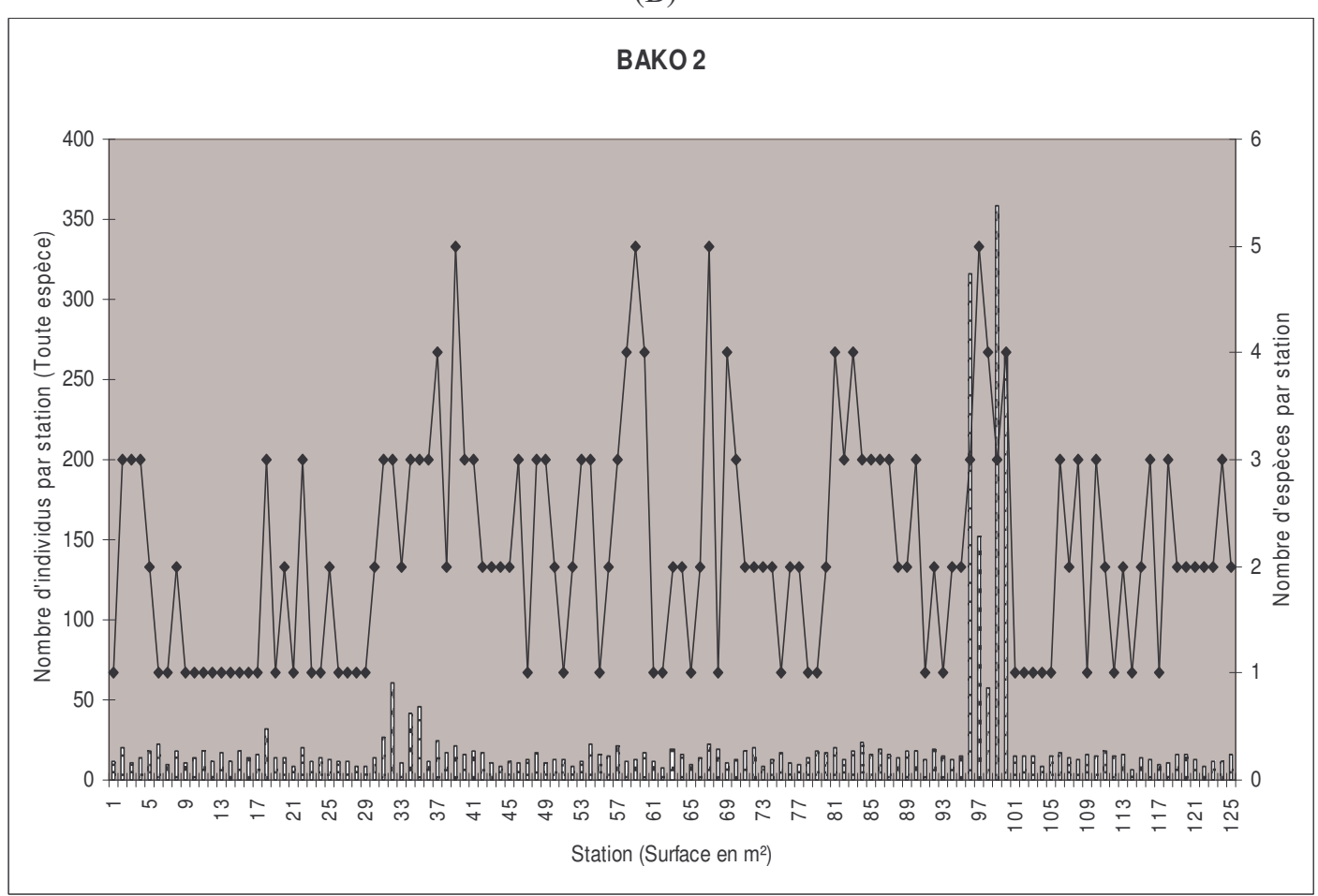

Figure 8 (A-B): Distribution de présence des espèces végétales dans les clairières de Bako 1 et Bako 2. 
(A)

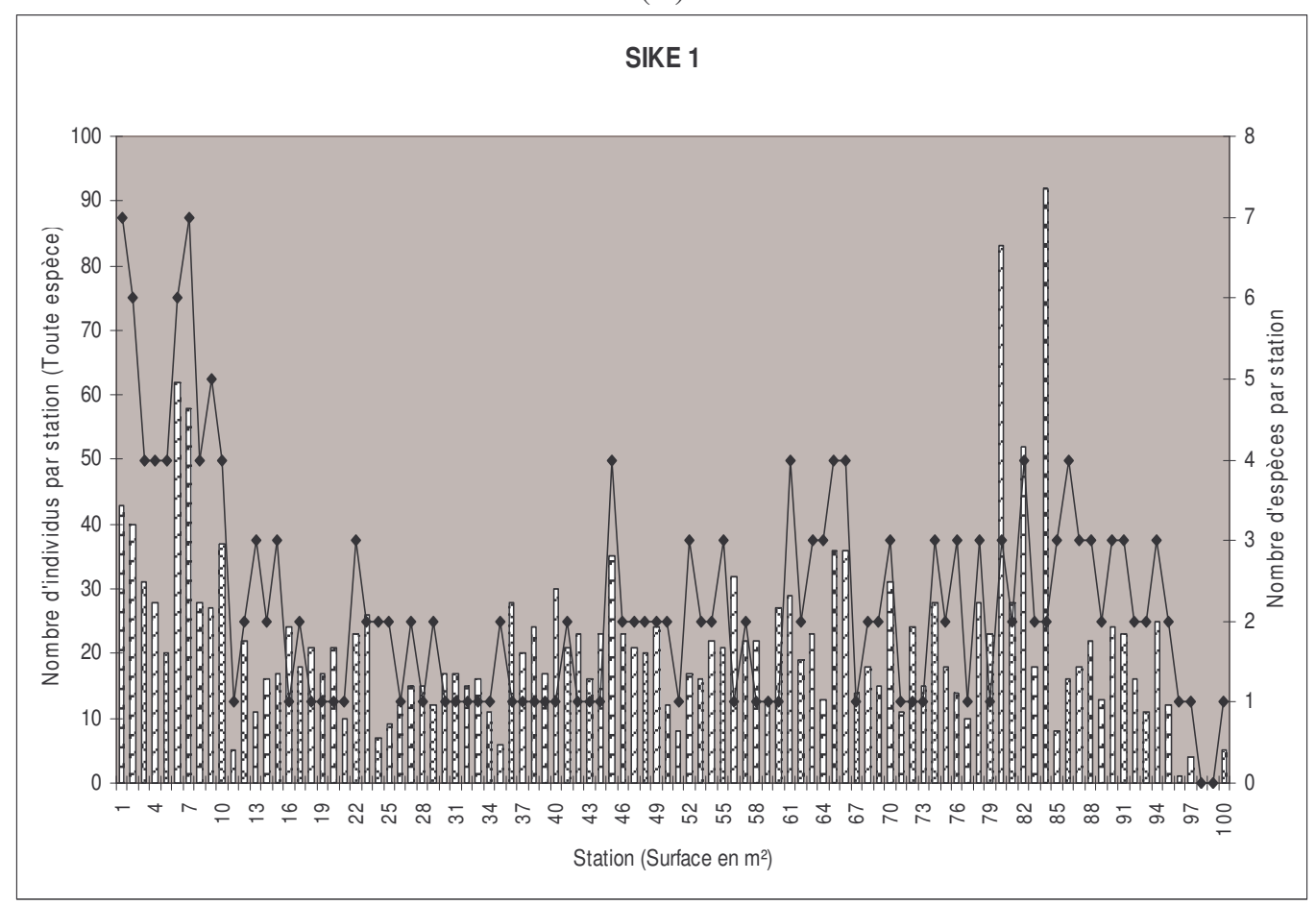

(B)

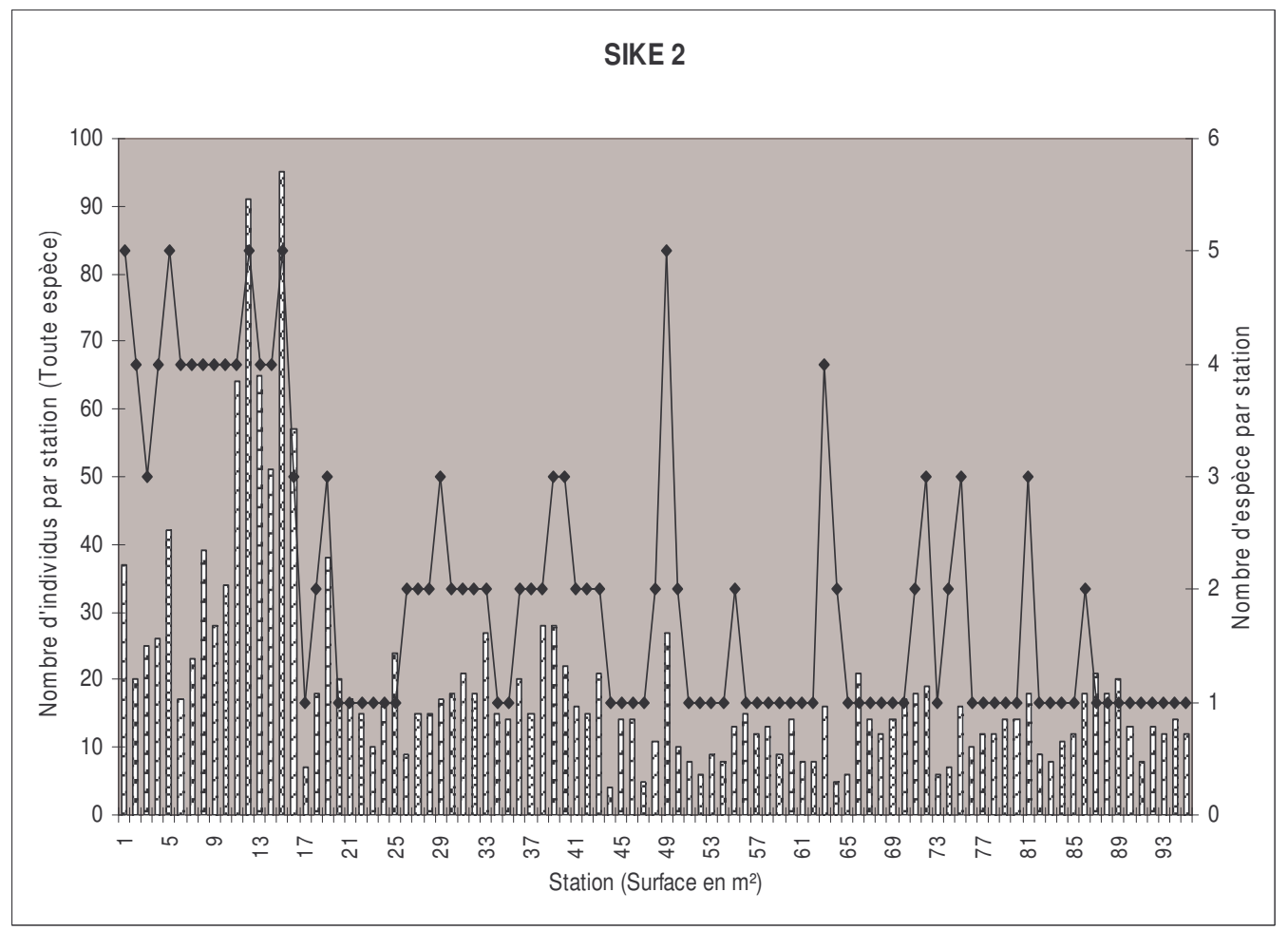

Figure 9 (A-B): Distribution de présence des espèces végétales dans les clairières de Sike 1 et Sike 2. 
(A)

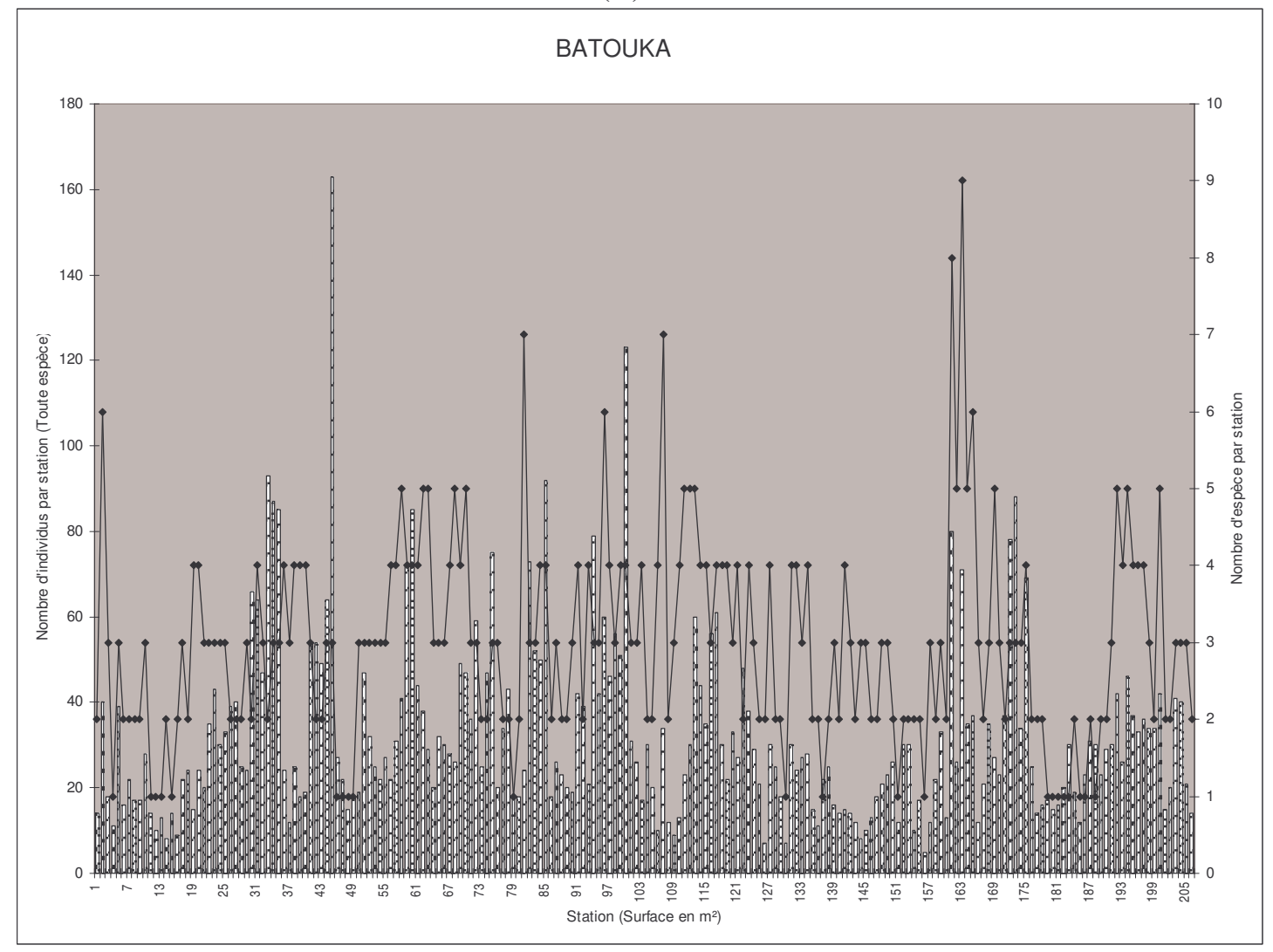

(B)

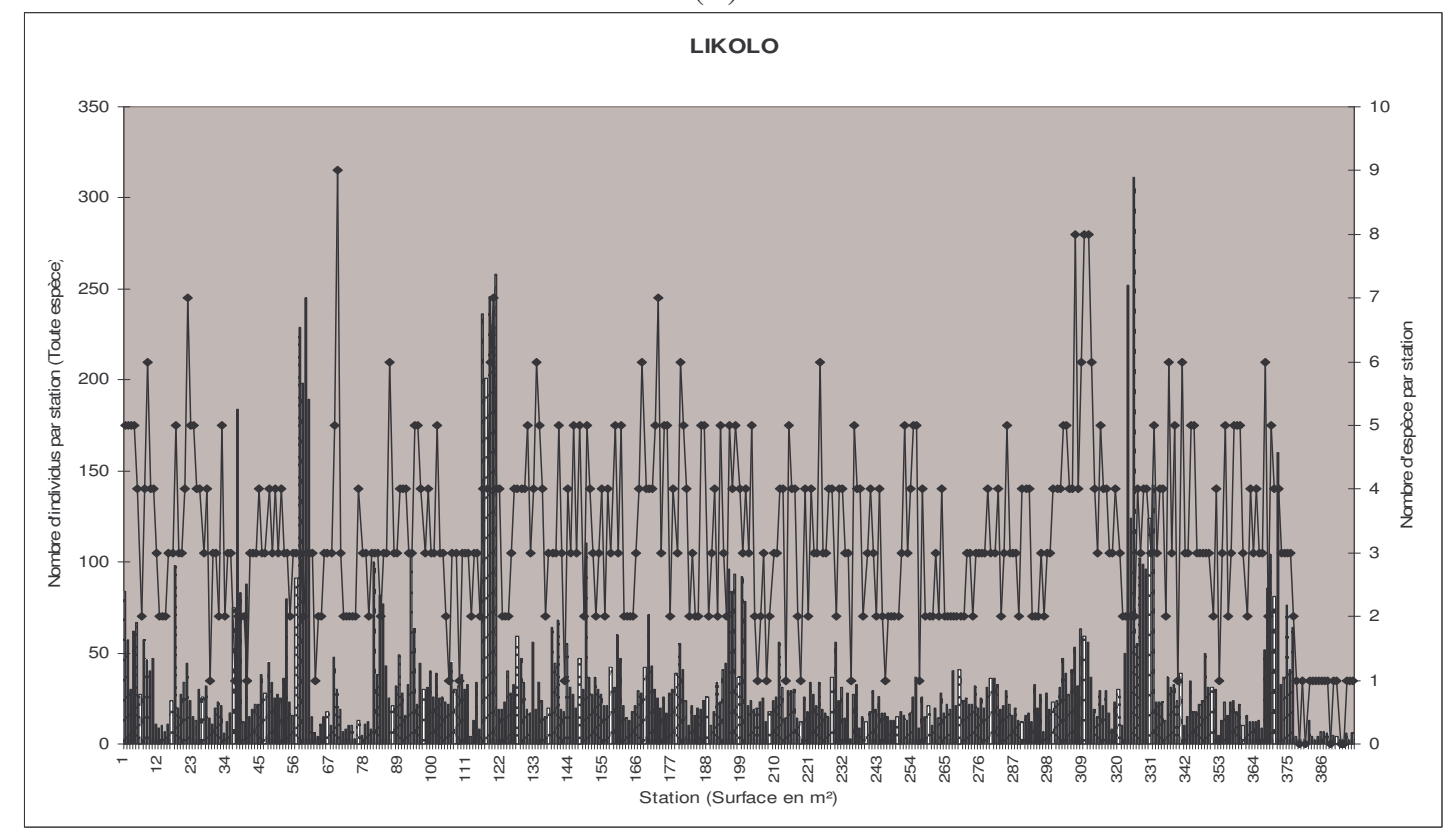

Figure 10 (A-B): Distribution de présence des espèces végétales dans les clairières de Batouka et Likolo. 


\section{DISCUSSION}

Cette étude a permis d'examiner la diversité biologique des clairières forestières dans le massif de Boumba-Bek qui est une unité représentative du massif forestier du Bassin du Congo. L'étude a montré que malgré leur apparente homogénéité, les clairières forestières sont porteuses d'une grande diversité floristique. Les clairières sur schiste ayant fait l'objet des inventaires floristiques ont affiché une diversité de 68 espèces d'herbacées, permettant ainsi une amélioration de la liste du cortège végétal de la région qui passe à 899 espèces appartenant à 137 familles en contribution aux travaux de Nkongmeneck (1996) et Ekobo (1998). Le peuplement graminoïde qui se compose de la famille des Poaceae et de celle des Cyperaceae représente 39,7\% d'espèces rencontrées dans les relevés réparties en Poaceae $(26,5 \%)$ et Cyperaceae $(13,2 \%)$ et 5 familles sur les 26 rencontrées (Poaceae, Cyperaceae, Fabaceae, Commelianceae et Malastomataceae) représentent $60,3 \%$ des espèces des relevés. Selon Descoings (1971), la proportion du peuplement graminoïde dans un relevé de la végétation est importante pour la qualité du pâturage. Noupa et Nkongmeneck (2008) ont montré que les clairières forestières étaient des sites de pâturage notamment pour les populations d'herbivores sauvage du massif forestier de Boumba-Bek. La richesse de ces écosystèmes en espèces de ces cinq familles dominantes justifie le caractère pastoral de ces milieux et leur importance pour la conservation des populations animales qui peuplent les forêts du Bassin du Congo. L'établissement d'un diagramme de distribution de présence/fréquence a permis de caractériser les milieux naturels en rendant compte de la richesse quantitative et qualitative du milieu traduit par la dominance ou l'abondance des espèces rencontrées. En permettant de déterminer la diversité quantitative et qualitative, le diagramme de distribution de présence/fréquence a permis de mettre en évidence les ressemblances physionomiques générales entre les clairières étudiées et les différences dans les relevés d'inventaire à l'échelle des placettes. L'étude a aussi permis de caractériser la végétation du milieu en termes d'abondance, de dominance et de fréquence spécifique. Ainsi, du point de vue de la dominance, L. simplex influence la physionomie des six clairières étudiées dans le massif de Boumba-Bek. Du point de vue de la dominance, l'étude montre que les clairières sont caractérisées par une abondance spécifique comprise entre 0 et 50 individus par relevé avec une moyenne inférieure à 20. Du point de vue de la fréquence, $L$. simplex est l'espèce végétale la plus fréquemment rencontrée des clairières étudiées.

Enfin, il se dégage de l'étude que les clairières sur schiste du massif de Boumba Bek sont plus diversifiées que riches. Ainsi, l'apparente homogénéité de la physionomie des clairières marquée parfois par la domination d'une seule espèce cache la réalité de la grande diversité floristique de ces écosystèmes.

\section{BIBLIOGRAPHIE}

Anonyme. 1990. Etat de la diversité biologique en République Démocratique du Congo. Annexes techniques. 17 p. http://bch-cbd.naturalsciences.be/congodr

Bene Bene CL. 2004. Monitoring of the illegal killing of elephants (MIKE). Inventaire en Afrique Centrale 20032004, site de Boumba-Bek (Cameroun). WWF Jengi SEFP, 49 p.

Boudet G. 1983. Systèmes de productions d'élevage au Sénégal. Etude du couvert herbacé. Compte rendu de fin d'étude. Maisons Alfort, IEMVT, $50 \mathrm{p}$.

Boudet G. 1991. Manuel sur les Pâturages Tropicaux et les Cultures Fourragères (5e édn). Maisons-Alfort: Institut d'Elevage et de Médecine Vétérinaire des Pays Tropicaux (IEMVT), (Manuels et Précis d'Elevage; 4); 266 p.

Boudet G, Meyer JP, Piot J, de Wispelaere G. 1980. Systèmes de productions d'élevage au Sénégal. Le couvert végétal et le cheptel. Rapport de campagne de recherche. Action concertée ACC/LAT/PVD, Maisons-Alfort, GERDAT/IEMVT, $12 \mathrm{p}$.

Braun-Blanquet. 1932. Plant sociology. The study of plant communities. New york, Conservation, Management and Sustainable Use. Occasional papers of 
the IUCN, Species Survival Commission $\mathrm{N}^{\circ} 6,242 \mathrm{p}$.

Descoings B. 1971. Méthode de description des formations herbeuses intertropicales par la structure de la végétation. Candollea, 26(2): 223-257.

Ekobo A. 1998. Large mammals and vegetation surveys in the Boumba-Bek and Nki forest project area. WWFCameroon, $123 \mathrm{p}$.

Encarta. 2000. Encyclopédie Microsoft Encarta.

Greig-Smith P. 1983. Quantitative Plant Ecology (3rd edn). Oxford: Blackwell; $298 \mathrm{p}$.

Harris D. 1999. Lobéké: botanical inventory. Report for WWF Cameroon, $130 \mathrm{p}$.

Letouzey R. 1969. Etude Phytogéographique du Cameroun. Le Chevalier: Paris; 513 p.
Letouzey R. 1985. Notice de la Carte Phytogéographique du Cameroun au 1/500.000. Institut de la Carte Internationale de la Végétation : Toulouse, France.

Mime. 2001. Rapport National sur la Diversité Biologique. République du Congo; $104 \mathrm{p}$.

Noupa P, Nzooh Dongmo Z-L, Fouda E-B. 2002. Evaluation préliminaire des potentialités fauniques et des activités anthropiques dans le massif forestier de ngoyla-mintom. WWF-Cameroon, $60 \mathrm{p}$.

Noupa P, Nkongmeneck B-A. 2008. Influence des clairières sur la répartition spatiale des grands mammifères dans la forêt dense du bassin du Congo: Cas du Parc National de Boumba-Bek (Sud-Est Cameroun). Int. J. Biol. Chem. Sci., 2(2): 185-195.

Nkongmeneck BA. 1996. The Boumba-Bek and Nki Forest Reserve, Botany and Ethnobotany. WWF Cameroon, 146 p. 